\title{
Time damping of non-adiabatic MHD waves in an unbounded partially ionised prominence plasma
}

\author{
P. Forteza, R. Oliver, and J. L. Ballester \\ Departament de Física, Universitat de les Illes Balears, 07122 Palma de Mallorca, Spain \\ e-mail: [pep. forteza; ramon.oliver; joseluis.ballester]@uib.es
}

Received 11 June 2008 / Accepted 12 September 2008

\begin{abstract}
Aims. We study the joint effect of ion-neutral collisions and thermal mechanisms on the damping of MHD waves in a partially ionised prominence plasma.

Methods. Thermal conduction, radiation, and heating were included in the energy equation of the one-fluid MHD set of equations we derived earlier for an adiabatic, partially ionised plasma. Then, assuming small perturbations, these equations were linearised and the dispersion relation for magnetoacoustic, Alfvén, and thermal waves obtained.

Results. Compared with the non-adiabatic, fully ionised, and the adiabatic, partially ionised cases, the main result is that there is an increase in the efficiency of the damping of magnetoacoustic waves in prominence oscillations within the observed range of wavelengths for the magnetoacoustic waves. On the other hand, the Alfvén wave is only damped by the ion-neutral collision mechanism, and this damping becomes important for almost neutral plasmas.

Conclusions. The coupling of non-adiabatic mechanisms with the ion-neutral collisions mechanism offers a more complete model for the damping of magnetoacoustic waves with those values compatible with those observed in prominence oscillations. Furthermore, the ion-neutral collisions mechanism is able to damp the Alfvén wave in an efficient way.
\end{abstract}

Key words. magnetohydrodynamics (MHD) - plasmas - waves - Sun: prominences

\section{Introduction}

Prominences are dense and relatively cool coronal structures that appear as thin dark ribbons on the solar disc or as bright features above the limb when observed in $\mathrm{H} \alpha$. These structures are supported against gravity by the magnetic field, which is also believed to be responsible forf the thermal isolation from the hotter corona.

Prominence material seems to be partially ionised, with an ionisation degree that varies over a wide range (Patsourakos \& Vial 2002). In general, models of prominences invoke a support mechanism for the prominence material consisting of an upward magnetic force, which balances the downward gravitational force. However, if the prominence plasma is only partially ionised, one might wonder how the neutral component of the plasma is supported against the gravity. For a plausible explanation of how neutrals are supported, we refer the reader to Gilbert et al. (2002).

There is strong observational evidence of small-amplitude oscillations in quiescent solar prominences. These oscillations have typical amplitudes going from less than $0.1 \mathrm{~km} \mathrm{~s}^{-1}$ to $2-3 \mathrm{~km} \mathrm{~s}^{-1}$, and they have been historically classified according to their periods in short- $(P<10 \mathrm{~min})$, intermediate- $(10 \mathrm{~min}<$ $P<40 \mathrm{~min}$ ), and long-period oscillations $(P>40 \mathrm{~min})$, although there are reported observations of very short-period of less than 1 min (Balthasar et al. 1993) and extremely ultra-longperiods of more than $8 \mathrm{~h}$ (Foullon et al. 2004). On the other hand, observations have also pointed out the damping of the oscillations in Doppler velocity time series, obtaining a damping time usually between 1 and 3 times the corresponding oscillation period (Molowny-Horas et al. 1999; Terradas et al. 2002).

Under prominence conditions, the plasma is described reasonably well by the equations of magnetohydrodynamics (MHD), so from the theoretical point of view, small-amplitude prominence oscillations can be interpreted in terms of linear MHD waves, whose attenuation has been studied by considering non-adiabatic effects, assuming radiative losses based on the Newtonian cooling with a constant relaxation time (Terradas et al. 2001) or by assuming a more complete treatment with the incorporation of optically thin radiation, heating, and thermal conduction (Carbonell et al. 2004, hereafter Paper I; Terradas et al. 2005). The main conclusion that arises from these works is that only the slow wave is damped by thermal effects in an efficient way, radiation being the dominant attenuation mechanism in the observed range of wavelengths; in contrast, the fast wave remains practically unaffected. On the other hand, Forteza et al. (2007), hereafter Paper II, proposed ion-neutral collisions as a damping mechanism of prominence oscillations on the basis that prominences, as noted previously, are partially ionised plasmas. This mechanism is efficient in attenuating the fast wave in plasmas with a small fraction of ions, while the slow wave is not affected. For more information about observations and theoretical models of solar prominence oscillations, we refer to various reviews on the topic (Oliver \& Ballester 2002; Wiehr 2004; Engvold 2004; Ballester 2006; Banerjee et al. 2007).

In this paper, our aim is to study the joint effect of ionneutral collisions and thermal mechanisms on the damping of MHD waves in a partially ionised plasma. The layout of this 
paper is as follows. In Sect. 2 the equations for a non-adibatic partially ionised plasma and the corresponding dispersion relation are presented. In Sect. 3 the results obtained by solving the dispersion relation and a comparison with Papers I and II are given. Finally, the main conclusions are summarised in Sect. 4.

\section{Basic equations}

In the equilibrium configuration, we consider a homogeneous hydrogen, prominence plasma $\left(p_{0}, \rho_{0}, T_{0}=\right.$ const. $)$ threaded by a uniform magnetic field along the $x$-direction, $\boldsymbol{B}_{0}=B_{0} \hat{\boldsymbol{x}}=$ const.

The basic equations for a non-adiabatic partially ionised plasma (Paper II), ignoring the effect of gravity, are

$\frac{\mathrm{d} \rho}{\mathrm{d} t}+\rho \nabla \cdot \boldsymbol{V}=0$

$\rho \frac{\mathrm{d} \boldsymbol{V}}{\mathrm{d} t}=-\nabla p+\frac{1}{4 \pi}(\nabla \times \boldsymbol{B}) \times \boldsymbol{B}$,

$\frac{\mathrm{d} p}{\mathrm{~d} t}-\frac{\gamma p}{\rho} \frac{\mathrm{d} \rho}{\mathrm{d} t}-\gamma \frac{\boldsymbol{j}}{e n_{\mathrm{i}}} \cdot \nabla p_{\mathrm{i}}=-(\gamma-1)[\rho L(\rho, T)-\nabla \cdot(\boldsymbol{\kappa} \cdot \nabla T)]$,

$$
\begin{aligned}
\frac{\partial \boldsymbol{B}}{\partial t}= & \nabla \times(\boldsymbol{V} \times \boldsymbol{B})+\eta \nabla^{2} \boldsymbol{B}-\Xi \nabla \times(\nabla p \times \boldsymbol{B}) \\
& +\frac{\eta_{\mathrm{C}}-\eta}{|\boldsymbol{B}|^{2}} \nabla \times\{[(\nabla \times \boldsymbol{B}) \times \boldsymbol{B}] \times \boldsymbol{B}\},
\end{aligned}
$$

$\nabla \cdot \boldsymbol{B}=0$,

$p=\frac{\rho R T}{\tilde{\mu}}$,

where $\mathrm{d} / \mathrm{d} t=\partial / \partial t+\boldsymbol{V} \cdot \nabla$ is the material derivative for time variations following the motion and $\Xi=\xi_{\mathrm{i}} \xi_{\mathrm{n}}^{2} /\left(1+\xi_{\mathrm{i}}\right) \alpha_{\mathrm{n}}$. Here, the variables $p, \rho, T$, and $\boldsymbol{B}$ have their usual meaning, while $n_{\mathrm{i}}$, $\boldsymbol{V}, \tilde{\mu}, \eta_{\mathrm{C}}, \xi_{\mathrm{i}}$, and $\xi_{\mathrm{n}}$ are the density of ions, the centre of mass velocity, the ionisation fraction, Cowling's magnetic resistivity, and the relative densities of ions and neutrals. Their definitions can be found in Paper II.

The inclusion of non-adiabatic effects modifies the energy equation, in which the thermal mechanisms (i.e., radiation, heating, and thermal conduction) are added, so we next concentrate on this equation and refer to Paper II for a detailed derivation of the MHD equations for an adiabatic partially ionised plasma and an in-depth study of the corresponding damping of the magnetoacoustic waves.

\subsection{Radiation and heating}

The heat-loss function represents the difference between an arbitrary heat input and radiative losses. In our case, we have chosen to represent these radiative losses by means of the optically thin radiative loss function (Hildner 1974). Then, our heat-loss function is given by

$L(\rho, T)=\frac{L_{\mathrm{r}}}{\rho}-h \rho^{a} T^{b}$.

The last term in Eq. (7) represents an arbitrary heating function that can be modified by taking different values for the exponents $a$ and $b$, while $L_{\mathrm{r}}$ represents radiative losses and can be written as (Priest 1984)

$L_{\mathrm{r}}=n_{\mathrm{e}} n_{\mathrm{H}} Q(T)$,
Table 1. Parameter values of the radiative loss function for prominence plasmas with different optical thicknesses, expressed in cgs units.

\begin{tabular}{lccl}
\hline \hline Regime & $\chi^{*}$ & $\alpha$ & Reference \\
\hline Prominence 1 & $1.76 \times 10^{-6}$ & 7.4 & Hildner (1974) \\
Prominence 2 & $1.76 \times 10^{-46}$ & 17.4 & Milne et al. (1979) \\
Prominence 3 & $7.01 \times 10^{-97}$ & 30 & Rosner et al. (1978) \\
\hline
\end{tabular}

where $n_{\mathrm{e}}$ is the electron density and $n_{\mathrm{H}}=n_{\mathrm{n}}+n_{\mathrm{i}}$ the density of hydrogen atoms and ions. The function $Q(T)$ has been evaluated by different authors (Cox \& Tucker 1969; Tucker \& Koren 1971; McWhirter et al. 1975; Raymond \& Smith 1977; Rosner et al. 1978) and can be approximated by $Q(T)=\chi T^{\alpha}, \chi$, and $\alpha$ being piecewise functions depending on the temperature (Hildner 1974).

After some simple calculations and taking into account that $\chi^{*}=\chi / m_{\mathrm{p}}^{2}$ and that the relative density of ions, $\xi_{\mathrm{i}}$, is defined as in Paper II $\left(\xi_{\mathrm{i}}=1 / \tilde{\mu}-1\right)$, the heat loss-function for a partially ionised plasma takes the form

$L(\rho, T)=\xi_{\mathrm{i}} \rho \chi^{*} T^{\alpha}-h \rho^{a} T^{b}$.

In the case of an equilibrium with uniform temperature, such as the one we consider here, the heat-loss function is

$L\left(\rho_{0}, T_{0}\right)=0$.

Of all the heating scenarios (Rosner et al. 1978; Dahlburg \& Mariska 1988), and taking into account that the different heating mechanisms do not affect the damping time in a significant way (Paper I), we have chosen a constant heating per unit volume $(a=b=0)$, so

$h=\xi_{\mathrm{i}} \rho_{0} \chi^{*} T_{0}^{\alpha}$.

The use of an optically thin radiative loss function seems to be a reasonable approach for coronal conditions, while it may not be valid for prominence conditions because they are optically thick at least in part. In prominences, the radiative losses from the internal part are greatly reduced, and this can be represented by changing the values of $\chi^{*}$ and $\alpha$ in the cooling function. The regimes considered in Table 1 reduce the losses by two orders of magnitude when we go from regime prominence 1 (Hildner 1974) to prominence 3 (Rosner et al. 1978).

\subsection{Thermal conduction}

The term $\nabla \cdot(\boldsymbol{\kappa} \cdot \nabla T)$ in the energy equation (Eq. (3)) represents thermal conduction, where $\kappa$ is the conductivity tensor that can be decomposed in its perpendicular and parallel components to the magnetic field,

$\boldsymbol{\kappa}=\kappa_{\|} \hat{\boldsymbol{b}} \hat{\boldsymbol{b}}+\kappa_{\perp}(\boldsymbol{I}-\hat{\boldsymbol{b}} \hat{\boldsymbol{b}})$,

where $\boldsymbol{I}$ is the identity tensor and $\hat{\boldsymbol{b}}$ the unitary vector in the direction of the magnetic field.

Following Parker (1953) and Ibáñez \& Mendoza (1990), the thermal conduction coefficient in a partially ionised plasma can be expressed as the sum of the contribution of the electrons and the contribution of the neutrals

$\kappa=\kappa_{\mathrm{e}}+\kappa_{\mathrm{n}}$.

For prominence applications the perpendicular component of the electron's contribution to the thermal conduction can be neglected, so

$\boldsymbol{\kappa}_{\mathrm{e}}=\kappa_{\mathrm{e} \|} \hat{\boldsymbol{b}} \hat{\boldsymbol{b}}$, 
with

$\kappa_{\mathrm{e} \|}=1.84 \times 10^{-5} \frac{\xi_{\mathrm{i}}}{\ln \Lambda_{\mathrm{C}}}$.

On the other hand, given that neutrals do not feel the magnetic field influence, their contribution to thermal conduction is isotropic, so

$\boldsymbol{\kappa}_{\mathrm{n}}=\kappa_{\mathrm{n}} \boldsymbol{I}$,

with

$\kappa_{\mathrm{n}}=2.5 \times 10^{3}\left(1-\xi_{\mathrm{i}}\right)$.

\subsection{Dispersion relation}

To obtain the dispersion relation for the MHD waves, we consider small perturbations from the equilibrium in the form

$$
\begin{aligned}
& \boldsymbol{B}(t, \boldsymbol{r})=\boldsymbol{B}_{0}+\boldsymbol{B}_{1}(t, \boldsymbol{r}), \quad p(t, \boldsymbol{r})=p_{0}+p_{1}(t, \boldsymbol{r}), \\
& \rho(t, \boldsymbol{r})=\rho_{0}+\rho_{1}(t, \boldsymbol{r}), \\
& \boldsymbol{V}(t, \boldsymbol{r})=\boldsymbol{V}_{1}(t, \boldsymbol{r}) .
\end{aligned}
$$

Then we linearise the basic Eqs. (1)-(6) and, since the medium is unbounded, we perform a Fourier analysis in plane waves and assume that perturbations behave as

$f_{1}(\boldsymbol{r}, t)=f \mathrm{e}^{i(\omega t+\boldsymbol{k} \cdot \boldsymbol{r})}$.

With no loss of generality, we choose the $z$-axis so that the wavevector $\boldsymbol{k}$ lies in the $x z$-plane $\left(\boldsymbol{k}=k_{x} \hat{\boldsymbol{x}}+k_{z} \hat{\boldsymbol{z}}\right)$. Then, the following scalar equations are obtained.

$\omega \rho_{1}+\rho_{0}\left(k_{x} V_{1 x}+k_{z} V_{1 z}\right)=0$,

$\omega \rho_{0} V_{1 x}+k_{x} p_{1}=0$

$\omega \rho_{0} V_{1 y}-\frac{B_{0 x}}{4 \pi} k_{x} B_{1 y}=0$,

$\omega \rho_{0} V_{1 z}+k_{z} p_{1}+\frac{B_{0 x}}{4 \pi}\left(k_{z} B_{1 x}-k_{x} B_{1 z}\right)=0$,

$i \omega\left(p_{1}-c_{\mathrm{s}}^{2} \rho_{1}\right)+(\gamma-1)\left(\kappa_{\mathrm{e} \|} k_{x}^{2}+\kappa_{\mathrm{n}} k^{2}+\rho 0 L_{T}\right) T_{1}$ $+(\gamma-1)\left(L+\rho_{0} L_{\rho}\right) \rho_{1}=0$

$B_{1 x}\left(i \omega+k_{x}^{2} \eta+k_{z}^{2} \eta_{\mathrm{C}}\right)+\left(\eta-\eta_{\mathrm{C}}\right) k_{x} k_{z} B_{1 z}$ $+B_{0 x} k_{z}\left(i V_{1 z}+k_{z} \Xi p_{1}\right)=0$,

$B_{1 y}\left(i \omega+k_{x}^{2} \eta_{\mathrm{C}}+k_{z}^{2} \eta\right)-i B_{0 x} k_{x} V_{1 y}=0$,

$B_{1 z}\left(i \omega+k_{x}^{2} \eta_{\mathrm{C}}+k_{z}^{2} \eta\right)+\left(\eta-\eta_{\mathrm{C}}\right) k_{x} k_{z} B_{1 x}$
$-B_{0 x} k_{x}\left(i V_{1 z}+k_{z} \Xi p_{1}\right)=0$

$k_{x} B_{1 x}+k_{z} B_{1 z}=0$,

$\frac{p_{1}}{p_{0}}-\frac{\rho_{1}}{\rho_{0}}-\frac{T_{1}}{T_{0}}=0$.

Equations (20) and (24) are decoupled from the rest and from them we can obtain the dispersion relation for Alfvén waves

$\omega^{2}-k_{x}^{2} \Gamma(\theta)^{2}=0$, where $\Gamma(\theta)$ is a modified and complex Alfvén speed $\left(\Gamma(\theta)=\Gamma_{\mathrm{R}}+\right.$ $i \Gamma_{\mathrm{I}}$ ) defined as

$\Gamma(\theta)^{2}=v_{\mathrm{a}}^{2}+i \omega\left(\eta_{\mathrm{C}}+\eta \tan ^{2} \theta\right)$.

From the rest of the equations and when imposing that the determinant of this algebraic system be zero, we obtain our general dispersion relation for thermal and magnetoacoustic waves,

$a_{5} \omega^{5}+a_{4} \omega^{4}+a_{3} \omega^{3}+a_{2} \omega^{2}+a_{1} \omega+a_{0}=0$,

whose coefficient are given by

$a_{0}=-\frac{i k^{2} k_{x}^{2} v_{\mathrm{a}}^{2}}{\rho_{0}}\left(A T_{0}-H \rho_{0}\right)$,

$a_{1}=k^{2}\left[c_{\mathrm{s}}^{2} v_{\mathrm{a}}^{2} k_{x}^{2}+\frac{\left(A T_{0}-H \rho_{0}\right) \Psi}{\rho_{0}}\right]$,

$a_{2}=i k^{2}\left[\frac{A T_{0}-H \rho_{0}}{\rho_{0}}+\frac{A T_{0} v_{\mathrm{a}}^{2}}{p_{0}}+c_{\mathrm{s}}^{2} \Psi\right]$,

$a_{3}=-k^{2}\left[\left(c_{\mathrm{s}}^{2}+v_{\mathrm{a}}^{2}\right)+\frac{A T_{0} \eta_{\mathrm{C}}}{p_{0}}\right]$,

$a_{4}=-i\left(\frac{A T_{0}}{p_{0}}+k^{2} \eta_{\mathrm{C}}\right)$,

$a_{5}=1$.

Here

$A=(\gamma-1)\left(\kappa_{\mathrm{e} \|} k_{x}^{2}+\kappa_{\mathrm{n}} k^{2}+\rho_{0} L_{T}\right)$

$H=(\gamma-1)\left(L+\rho_{0} L_{\rho}\right)$,

$\Psi=k^{2} \eta_{\mathrm{C}}-k_{z}^{2} v_{\mathrm{a}}^{2} \Xi \rho_{0}$,

and $L_{\rho}$ and $L_{T}$ are defined as in Paper I. Using the propagation angle, $\theta$, between $\boldsymbol{k}$ and $\boldsymbol{B}_{0}$, the wavenumber components can be expressed as $k_{x}=k \cos \theta$ and $k_{z}=k \cos \theta$.

This dispersion relation is consistent with the limit cases studied in previous works. We can obtain the dispersion relation for non-adiabatic, magnetohydrodynamic waves (Paper I) by making the plasma fully ionised, $\Xi=0$, and assuming infinite conductivity, $\eta_{\mathrm{C}}=\eta=0$. In a similar way, one can obtain the dispersion relation for adiabatic partially ionised plasmas (Paper II) by imposing $A=H=0$.

Soler et al. (2007) introduces a modified sound speed for the pure non-adiabatic case which, using Eq. (22), can be extended to the case of partial ionisation. This modified and complex sound speed, $\Lambda=\Lambda_{\mathrm{R}}+i \Lambda_{\mathrm{I}}$, is given by

$\Lambda^{2}=\frac{\frac{T_{0}}{\rho_{0}} A-H+i c_{\mathrm{s}}^{2} \omega}{\frac{T_{0}}{\rho_{0}} A+i \omega}$.

Using this modified sound speed the magnetoacoustic waves dispersion relation can be written in a more compact form as

$$
\begin{array}{r}
\left(\omega^{2}-k^{2} \Lambda^{2}\right)\left(i k^{2} \eta_{\mathrm{C}} \omega-\omega^{2}\right)+k^{2} v_{\mathrm{a}}^{2}\left(\omega^{2}-k_{x}^{2} \Lambda^{2}\right) \\
+i k^{2} k_{z}^{2} v_{\mathrm{a}}^{2} \Lambda^{2} \Xi \rho_{0} \omega=0 .
\end{array}
$$



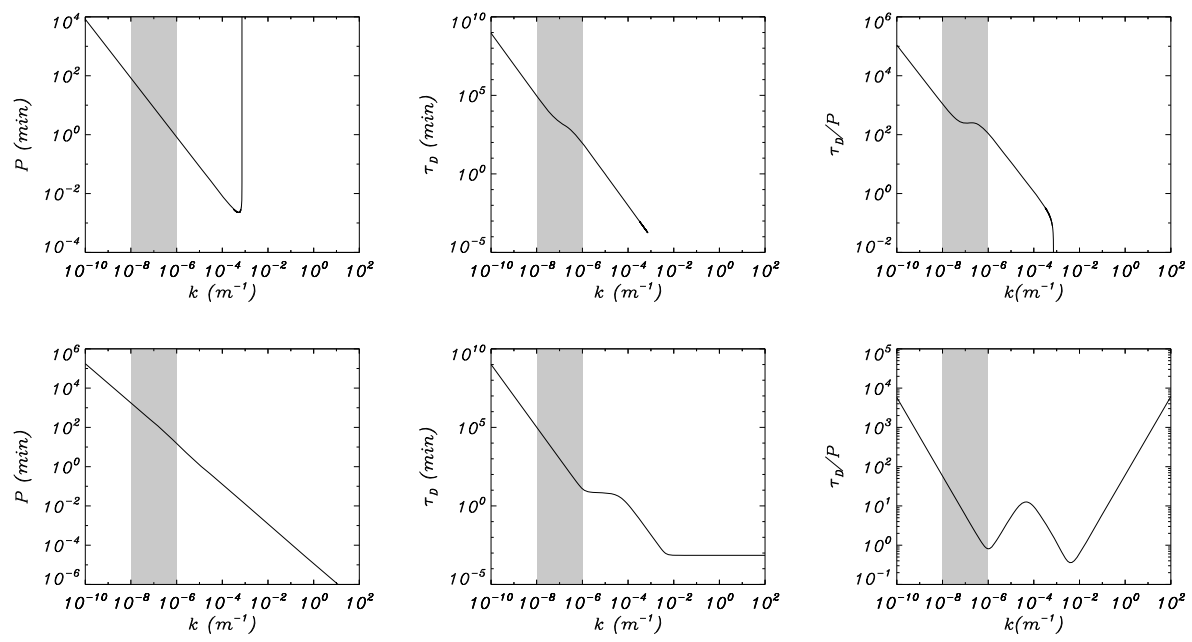

Fig. 1. Period, damping time, and ratio of the damping time to the period as a function of wavenumber $\boldsymbol{k}$ for fast (top) and slow (bottom) waves in a non-adiabatic partially ionised plasma with $\tilde{\mu}=0.8$. The shade region corresponds to the interval of observed wavelengths in prominences.

\section{Results}

\subsection{Magnetoacoustic waves}

We have numerically solved the dispersion relation (Eq. (30)) using symbolic software and considering a magnetic field $B_{0}=$ $10 \mathrm{G}$ and a propagation angle $\theta=\pi / 4$. Since we are interested in the temporal damping of magnetoacoustic waves, we consider the wavenumber, $k$, to be real and seek complex solutions of the frequency $\omega$ expressed as $\omega=\omega_{\mathrm{R}}+i \omega_{\mathrm{I}}$. Then, the period and damping time of the waves can be calculated as $P=2 \pi / \omega_{\mathrm{R}}$ and $\tau_{\mathrm{D}}=1 / \omega_{\mathrm{I}}$. We also calculate the ratio of the damping time to the period, $\tau_{\mathrm{D}} / P$, in order to compare the model with observations, which indicate that this parameter takes values around 3 .

The values of the density and temperature in the equilibrium configuration have been taken as $\rho_{0}=5 \times 10^{-14} \mathrm{~g} / \mathrm{cm}^{3}$ and $T_{0}=8000 \mathrm{~K}$. Unless otherwise stated, for the radiative loss function, we use the parameter values corresponding to prominence 1 regime in Table 1.

Figure 1 shows the results obtained for $P, \tau_{\mathrm{D}}$, and $\tau_{\mathrm{D}} / P$ corresponding to fast and slow waves. The shaded region defines the interval of observed wavelengths in prominence oscillations, which corresponds to the wavenumber interval $k=10^{-8}$ to $10^{-6} \mathrm{~m}^{-1}$. The thermal mode does not correspond to a propagating wave $\left(\omega_{R}=0\right)$, and its behaviour is quite similar to that of the non-adiabatic fully ionised case. For this reason, it is not considered in this work.

The slow mode curves qualitatively resemble those obtained in Paper I, but perhaps the most interesting feature is that the fast wave disappears at a certain wavenumber $\left(\sim 7 \times 10^{-4} \mathrm{~m}^{-1}\right)$. In order to understand this effect, let us consider the dispersion relation Eq. (35) for parallel propagation $\left(k_{z}=0, k=k_{x}\right)$,

$$
\left(\omega^{2}-k_{x}^{2} \Gamma(0)^{2}\right)\left(\omega^{2}-k_{x}^{2} \Lambda^{2}\right)=0,
$$

so that the fast wave is decoupled from the thermal and slow waves and its frequency is obtained by imposing that the first factor of the previous equation vanishes. Then, solving this dispersion relation for $\omega$ we obtain

$\omega=\frac{i k^{2} \eta_{\mathrm{C}}}{2} \pm \frac{k}{2} \sqrt{4 v_{\mathrm{a}}^{2}-k^{2} \eta_{\mathrm{C}}^{2}}$

Then, to have $\omega_{\mathrm{R}} \neq 0,4 v_{\mathrm{a}}^{2}-k^{2} \eta_{\mathrm{C}}^{2}$ must be greater than zero, which leads to

$k<\frac{2 v_{\mathrm{a}}}{\eta_{\mathrm{C}}} \equiv k_{\mathrm{c}}$
Hence, in a partially ionised plasma the fast mode only exists as a damped propagating wave for wavenumbers below the critical value, $k_{\mathrm{c}}$. For wavenumbers greater than this critical value, we have a damped disturbance instead of a propagating wave.

Using the parameter values mentioned before we obtain $k_{\mathrm{c}} \sim$ $7.3 \times 10^{-4} \mathrm{~m}^{-1}$, very similar to the value $\sim 7 \times 10^{-4} \mathrm{~m}^{-1}$ derived from Fig. 1. Given that Eq. (37) has been derived using adiabatic conditions and $\theta=0$, this agreement indicates that non-adiabatic effects and non-parallel propagation do not produce a substantial modification of $k_{\mathrm{c}}$.

\subsubsection{Effect of the ionisation degree}

Now, we study the effect of the ionisation fraction on the magnetoacoustic waves period and damping time. Figure 2 shows the results for fast and slow waves for four different values of the ionisation fraction going from fully ionised plasma $(\tilde{\mu}=0.5)$ to almost neutral plasma $(\tilde{\mu}=0.99)$. In the case of fast waves we observe that, for a fixed wavenumber, the damping time decreases when the ionisation degree is decreased, in agreement with Paper II. Moreover, the curve representing the period of the fast wave stops at different critical wavenumbers, $k_{\mathrm{c}}$, when the ionisation fraction is modified. The reason is that the critical wavenumber (Eq. (37) and Fig. 3) depends on the ionisation fraction through $\eta_{\mathrm{C}}$ (see Paper II for more details).

In the case of slow waves, the behaviour is more complex. First, the minimum of $\tau_{\mathrm{D}} / P$ at long wavelengths suffers a displacement towards longer wavelengths when $\tilde{\mu}$ is increased, and when $\tilde{\mu}>0.8$ it is located within the interval of observed wavelengths in prominence oscillations. This means that a higher attenuation efficiency is obtained for lower ionisation degrees. In addition, the minimum of $\tau_{\mathrm{D}} / P$ at short wavelengths displays two different features. On one hand, such as happens with the other minimum, there is a displacement towards longer wavelengths, although it is less pronounced. On the other hand, for $\tilde{\mu}>0.8$ this minimum splits into two different minima that become more separated as $\tilde{\mu}$ is increased. The presence of a new minimum in $\tau_{\mathrm{D}} / P$ yields the possibility of achieving very high damping rates for three wavelength ranges centred about the three minima.

\subsubsection{Effect of damping mechanisms}

In this section we assess the influence of the different damping mechanisms on the ratio of the damping time to the 

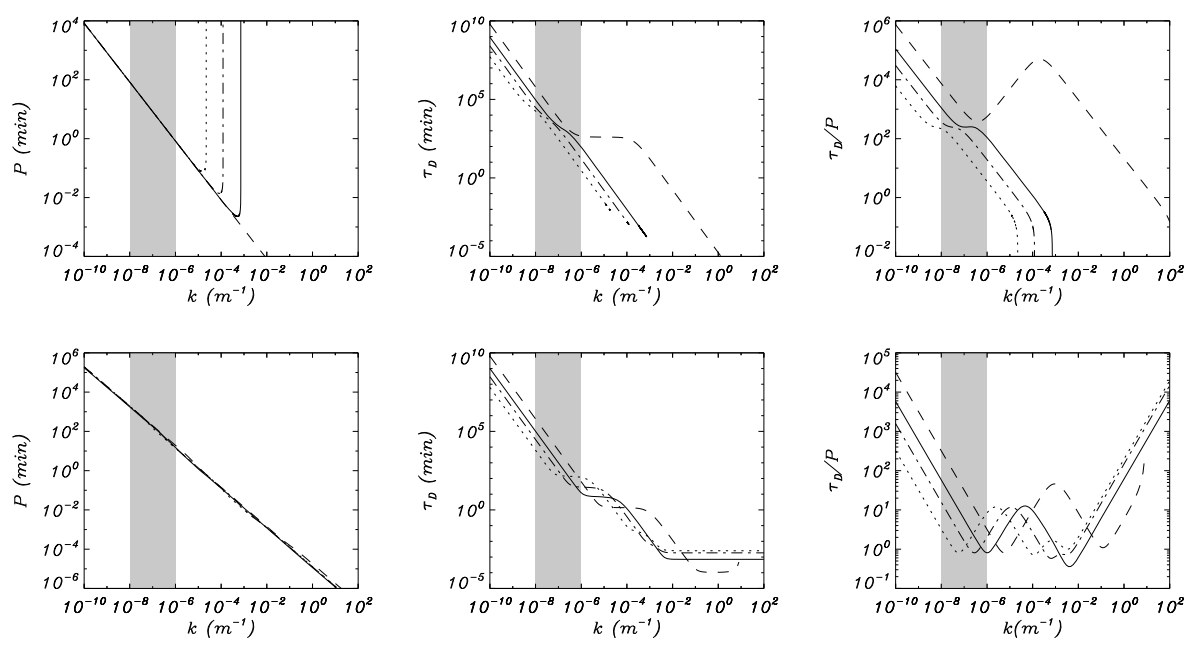

Fig. 2. Period, damping time, and ratio of the damping time to the period as a function of wavenumber $\boldsymbol{k}$ for the fast (top) and slow (bottom) waves for different ionisation fractions: $\tilde{\mu}=0.5$ (dashed), $\tilde{\mu}=0.8$ (solid), $\tilde{\mu}=0.95$ (dash-dotted), and $\tilde{\mu}=0.99$ (dotted). The shade region corresponds to the interval of observed wavelengths in prominences.

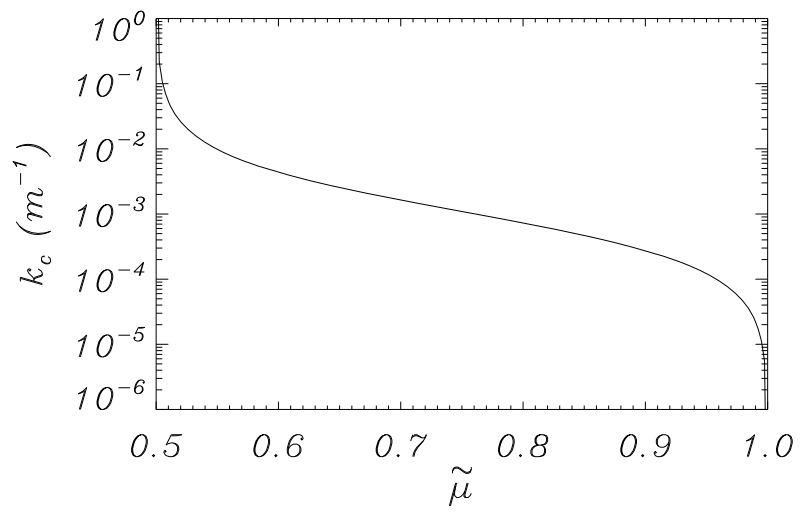

Fig. 3. Variation of the fast wave critical wavenumber, $k_{\mathrm{c}}$, versus the ionisation fraction, $\tilde{\mu}$.

period $\left(\tau_{\mathrm{D}} / P\right)$. First of all, we focus on the fast wave, and according to the top left panel of Fig. 4 (corresponding to $\tilde{\mu}=0.8$ ), the wavenumber interval considered can be divided in two regions with different dominant damping mechanisms. For small wavenumbers, i.e. long wavelengths, the ratio of the damping time to the period is dominated by radiative cooling (according to Paper I thermal conduction is negligible for small wavenumbers compared to radiative cooling), while it is dominated by ion-neutral collisions for large wavenumbers. This behaviour is similar for $\tilde{\mu}=0.99$, but the wavenumber at which the dominant mechanism changes is larger. Thermal conduction by neutrals and electrons does not influence the damping of the fast wave in a significant way.

The slow wave presents a more complex behaviour and we consider the two different values of the ionisation fraction separately. For almost neutral plasmas $(\tilde{\mu}=0.99)$, the ratio of the damping time to the period presents three minima of maximum attenuation, each of them corresponding to a different dominant damping mechanism. The first one, situated at long wavelengths, is caused by radiative cooling (see Paper I); the second one, in the mid range of the wavenumber interval, is due to ion-neutral collisions mechanisms; and finally, the last peak, corresponding to short wavelengths, is produced by neutral thermal conduction. As mentioned in Sect. 2.2, the expression of $\boldsymbol{\kappa}$ has two terms in a partially ionised plasma(Eq. (12)), corresponding to the contributions of neutrals and electrons. For a typical prominence temperature, the contribution of electrons to thermal conduction is negligible compared that of neutrals. In Fig. 4, the curve corresponding to the neutrals contribution to the thermal conduction is indistinguishable from the curve considering the joint contribution of neutrals and electrons.

For a higher ionisation fraction $(\tilde{\mu}=0.8$; bottom left panel of Fig. 4), the separation between the minima caused by ionneutral collisions and thermal conduction decreases. Because of this, the two minima merge and both effects are important in the same region of the considered wavenumber interval.

\subsubsection{Comparison with Carbonell et al. (2004) and Forteza} et al. (2007)

Next, we compare our results with previous ones in which nonadiabatic (Paper I) and partially ionised (Paper II) effects were considered separately. Figure 5 shows the period, damping time, and the ratio of the damping time to the period of the magnetoacoustic waves for three different cases: adiabatic partially ionised plasma, non-adiabatic fully ionised plasma, and non-adiabatic partially ionised plasma. We can observe that the wavenumber at which the fast wave disappears is not affected by the nonadiabatic terms because it arises from magnetic diffusion and is determined by partial ionisation effects. On the other hand, nonadiabatic and partial ionisation effects affect the period of the waves only slightly, which remains basically the same as in the ideal case. In Sect. 3.1.2 there is an explanation of the behaviour of the two minima of $\tau_{\mathrm{D}} / P$ of the slow wave.

Also, when comparing the three scenarios we observe that, in the non-adiabatic partially ionised case, there is an increase in the efficiency of the damping of fast and slow waves in the observed wavelength range.

Now, we can compare the value of the real part of the modified sound speed, Eq. (34), for the non-adiabatic partially and fully ionised plamas (Fig. 6). For the two ionisation degrees considered, $\Lambda_{R}$ has the same behaviour but suffers a displacement towards smaller wavenumbers and the size of the top flat region, where $\Lambda_{\mathrm{R}} \sim c_{\mathrm{s}}$, is increased when the ionisation fraction is increased. The different values of the height of the top flat region of the real part of the modified sound speed stem from the adiabatic sound speed, $c_{\mathrm{s}}$, depending on the ionisation fraction (Paper II).

We have studied the differences in the behaviour of the magnetoacoustic waves produced by the change of the parameters $\chi^{*}$ and $\alpha$ corresponding to the different prominence regimes. The results are very similar to those of Paper I for a fully ionised plasma, and the only difference is that the different prominence regimes only affect the region of the wavenumber 

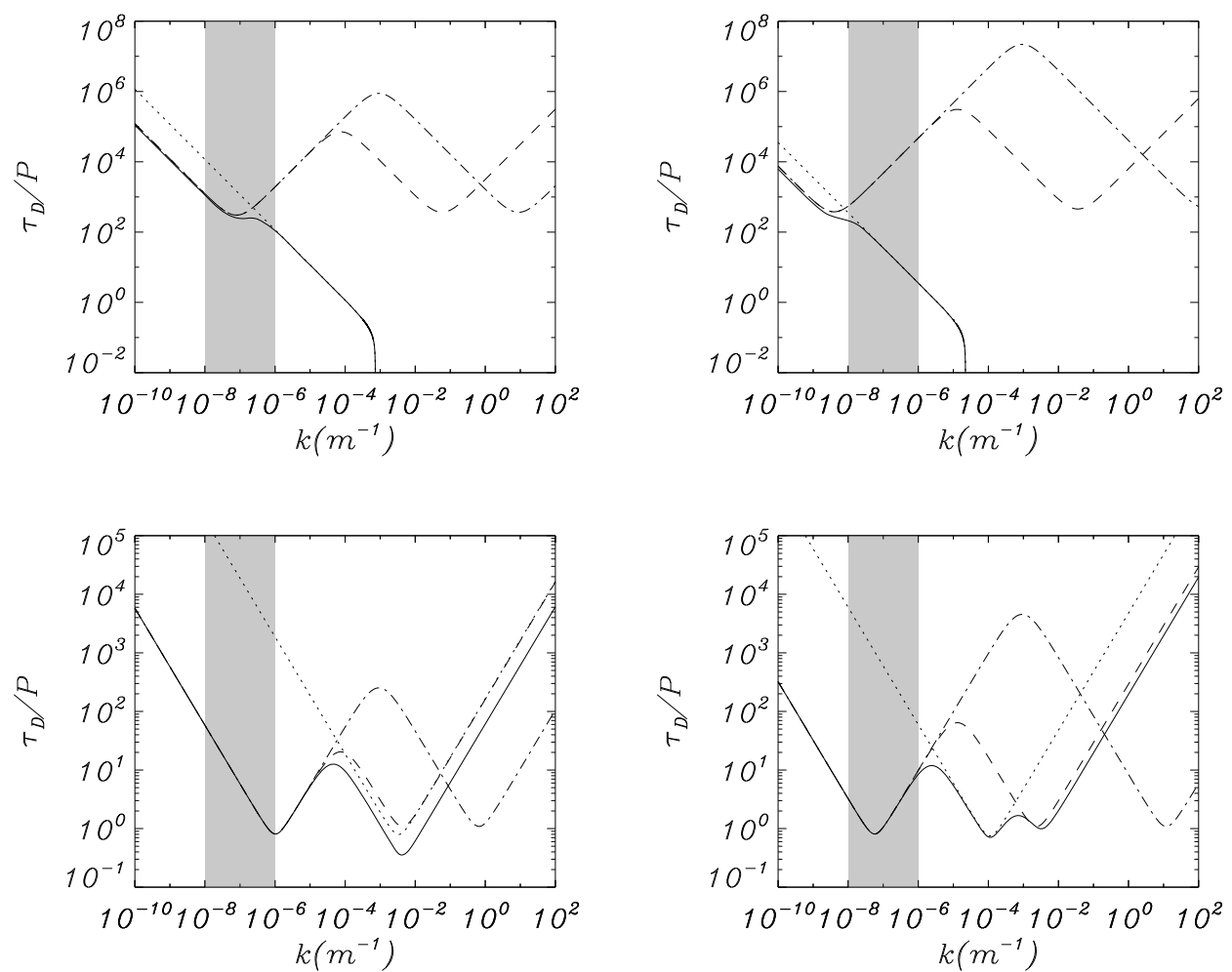

Fig. 4. Ratio of the damping time to the period as a function of wavenumber $\boldsymbol{k}$ for fast (top) and slow (bottom) waves corresponding to different ionisation fractions, $\tilde{\mu}=0.8$ (left) and $\tilde{\mu}=0.99$ (right). Different line styles correspond to: ion neutral collisions plus thermal mechanisms (solid line), only ion-neutral collisions (dotted line), only thermal mechanisms (dashed line), and only radiation, heating, and electronic thermal conduction (dash-dotted line). The shade region corresponds to the interval of observed wavelengths in prominences.
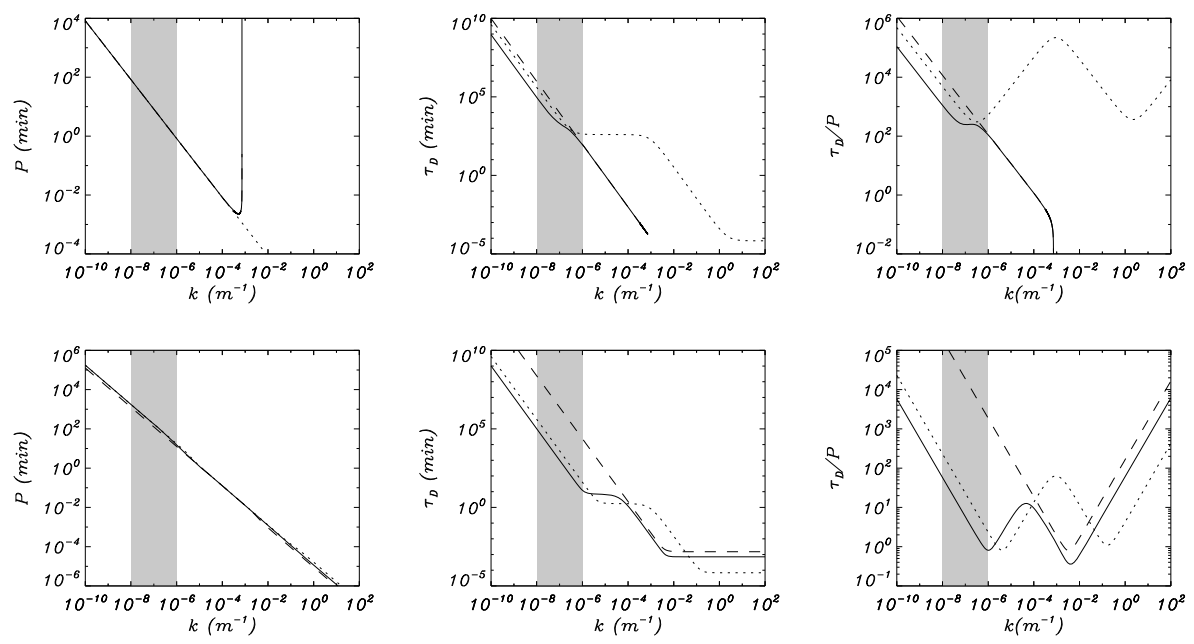

Fig. 5. Period, damping time, and ratio of the damping time to the period as a function of wavenumber $\boldsymbol{k}$ for the fast (top) and slow (bottom) waves. Solid lines: non-adiabatic partially ionised plasma; dashed lines: adiabatic partially ionised plasma; dotted lines: nonadiabatic fully ionised plasma. The shaded region corresponds to the interval of observed wavelengths in prominences.
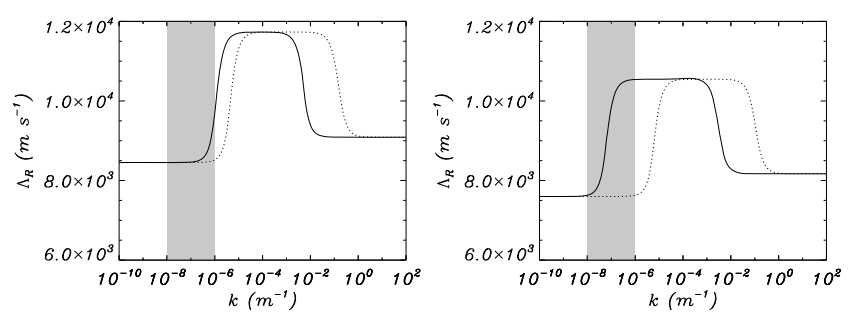

Fig. 6. Comparison of the real part of modified sound speed as a function of wavenumber $\boldsymbol{k}$ in a non-adiabatic partially ionised plasma (solid) and in a non-adiabatic fully ionised plasma (dotted) with $\tilde{\mu}=0.8$ (left) and $\tilde{\mu}=0.99$ (right). The two dotted curves are different because $c_{\mathrm{s}}$ depends on $\tilde{\mu}$. The shade region corresponds to the interval of observed wavelengths in prominences. interval in which thermal mechanisms are the dominant damping mechanisms.

\subsubsection{Dependence on the propagation angle}

Figure 7 shows the ratio of the damping time to the period for different propagation angles and the three different scenarios considered in Sect. 3.1.3: non-adiabatic fully ionised plasma (Paper I), adiabatic partially ionised plasma (Paper II) and nonadiabatic partially ionised plasma (present work).

For the non-adiabatic fully ionised plasma (left panels), the damping of the slow wave presents a weak dependence on the propagation angle, while the damping of the fast wave is strongly affected by the value of the propagation angle 

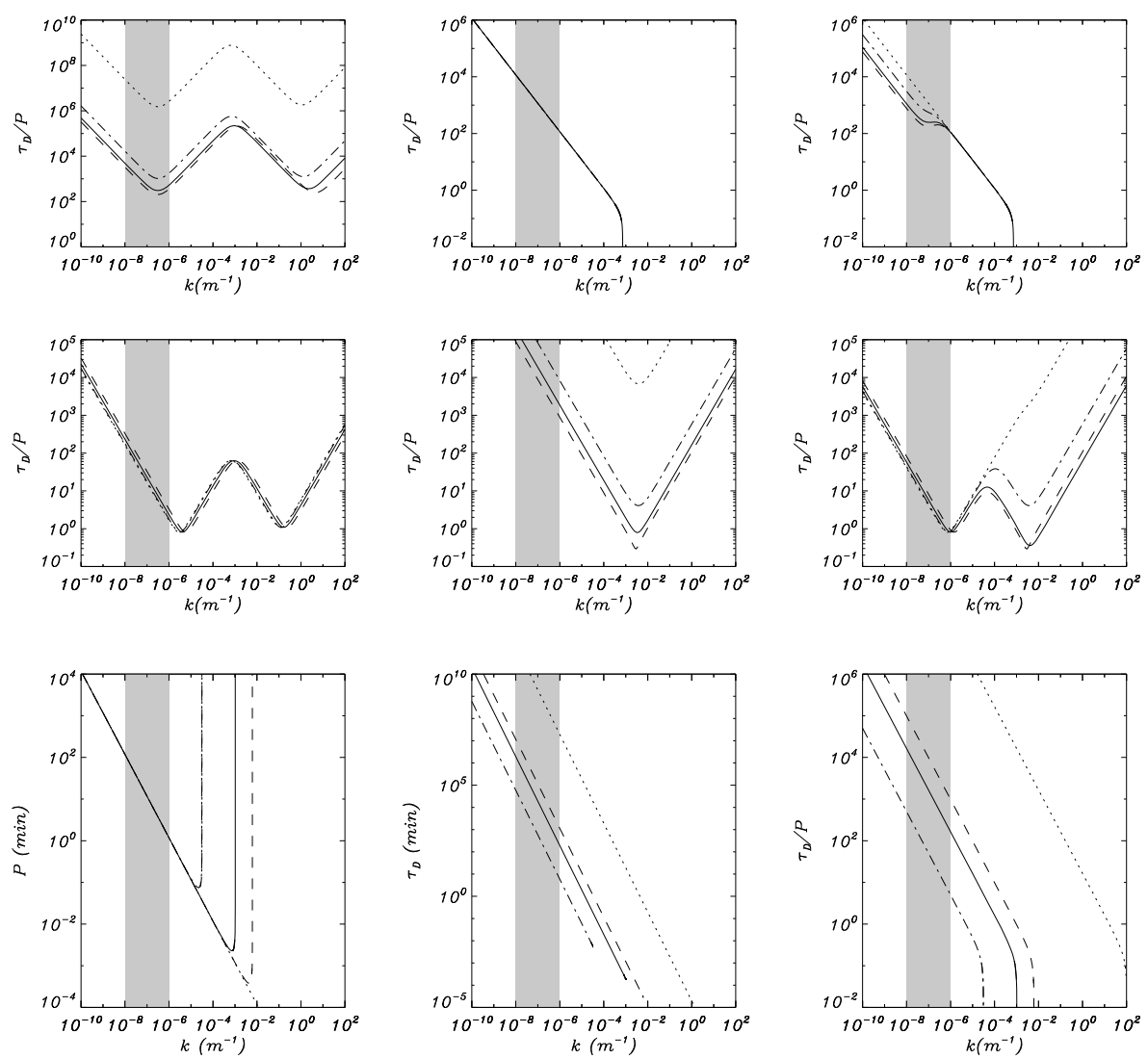

Fig. 7. Ratio of the damping time to the period as a function of wavenumber $\boldsymbol{k}$ for three different scenarios: non-adiabatic fully ionised plasma (left), adiabatic partially ionised plasma (centre), and non-adiabatic partially ionised plasma (right). The top panels correspond to the fast wave, while the bottom ones correspond to the slow wave. Different propagation angles have been considered: $\theta=0.01$ (dotted), $\theta=\pi / 8$ (dash-dotted), $\theta=\pi / 4$ (solid), and $\theta=\pi / 3$ (dashed). The shade region corresponds to the interval of observed wavelengths in prominences.

Fig. 8. Period, damping time, and ratio of the damping time to the period as a function of wavenumber $\boldsymbol{k}$ for the Alfvén wave in a nonadiabatic partially ionised plasma with $\tilde{\mu}=0.5$ (dotted), $\tilde{\mu}=0.6$ (dashed), $\tilde{\mu}=0.8$ (solid), and $\tilde{\mu}=0.99$ (dash-dotted). The shade region corresponds to the interval of observed wavelengths in prominences.
(Carbonell et al. 2006). This behaviour is reversed in an adiabatic partially ionised plasma: the slow wave is considerably affected, while the fast wave remains unaffected (centre panels).

In the non-adiabatic partially ionised case (right panels), both waves are notably affected by the variation of the propagation angle. Fast waves present a dependence on the propagation angle only in the wavenumber interval in which non-adiabatic effects are the dominant damping mechanisms. Meanwhile, for slow waves, the dependence on the propagation angle is stronger in the region in which ion-neutral collisions and thermal conduction are important. We can observe that for large propagation angles, the minimum caused by ion-neutral collisions is more pronounced, while this minimum completely disappears for small angles.

\subsection{Alfvén waves}

From the dispersion relation, Eq. (28), we know that the Alfvén wave is not influenced by the non-adiabatic terms and that it is only affected by the ion-neutral collisions terms. Since $\Gamma$ is a complex quantity, the real and imaginary parts of the Alfvén frequency are given by

$\omega_{\mathrm{R}}= \pm k_{x} \Gamma_{\mathrm{R}}$,

and

$\omega_{\mathrm{I}}= \pm k_{x} \Gamma_{\mathrm{I}}$.

Figure 8 shows the results obtained for the period, the damping time, and the ratio of the damping time to the period. In this figure the solution for a fully ionised plasma $(\tilde{\mu}=0.5)$ with magnetic resistivity (Ferraro \& Plumpton 1961; Kendall \& Plumpton 1964) is also shown.
The Alfvén wave behaviour is similar to that of the fast wave in the adiabatic partially ionised case, although in the nonadiabatic partially ionised case, both waves have a different behaviour in the wavenumber interval in which the behaviour of the fast wave is dominated by radiative losses. When ion-neutral collisions become the dominant mechanism, fast and Alfvén waves have similar period and damping times and, like for the fast wave, the ratio of the damping time to the period decreases when going to almost neutral plasmas. Also, from Fig. 9 one can conclude that the frequency of the Alfvén wave depends slightly on the angle of propagation.

On the other hand, from Eq. (28) we can obtain the critical wavenumber for the Alfvén waves, which is

$$
k_{\mathrm{c}}^{\mathrm{a}}=\frac{2 v_{\mathrm{a}}}{\cos \theta\left(\eta_{\mathrm{C}}+\eta \tan ^{2} \theta\right)} .
$$

This quantity depends on the ionisation fraction (as the fast wave critical wavenumber does) and on the propagation angle (Fig. 10). Usually, $k_{\mathrm{c}}^{\mathrm{a}}$ is bigger than $k_{\mathrm{c}}$ and both critical wavenumbers become equal for parallel propagation. On the other hand, when we consider $\eta_{\mathrm{C}}=\eta$, we recover from Eq. (38) the critical wavenumber of fully ionised and resistive plasmas (Ferraro \& Plumpton 1961; Kendall \& Plumpton 1964), which is given by

$k_{\mathrm{c}}^{\mathrm{FP}}=\frac{2 v_{\mathrm{a}}}{\eta} \cos \theta$

The ratio between the critical wavenumber for a partially ionised plasma, Eq. (38), and that of a fully ionised resistive plasma, Eq. (39) is given by

$\frac{k_{\mathrm{c}}^{\mathrm{FP}}}{k_{\mathrm{c}}^{\mathrm{a}}}=\sin ^{2} \theta+\frac{\eta_{\mathrm{C}}}{\eta} \cos ^{2} \theta$, 

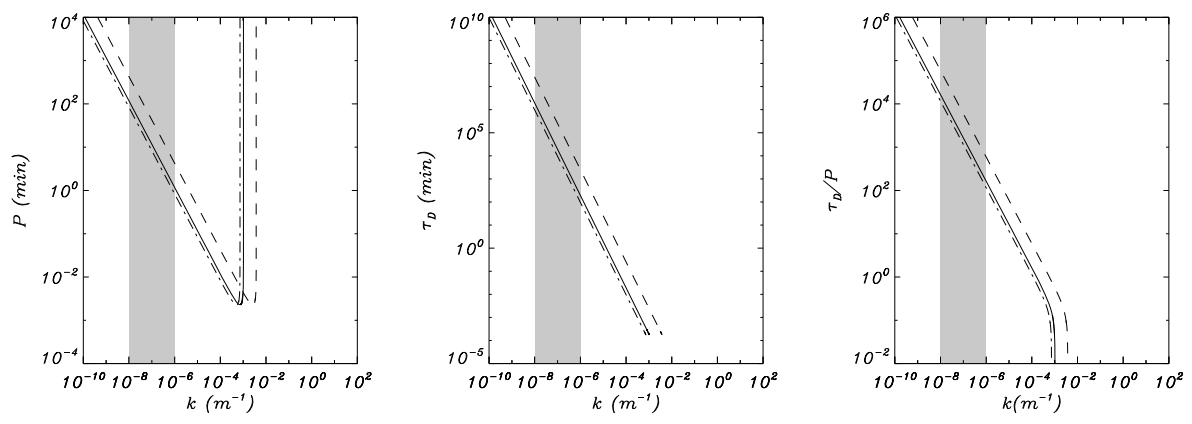

Fig. 9. Period, damping time, and ratio of the damping time to the period as a function of wavenumber $\boldsymbol{k}$ for the Alfvén wave in a nonadiabatic partially ionised plasma with $\theta=$ 0 (dash-dotted), $\theta=\pi / 4$ (solid), and $\theta=$ $7 \pi / 16$ (dashed). The shade region corresponds to the interval of observed wavelengths in prominences.

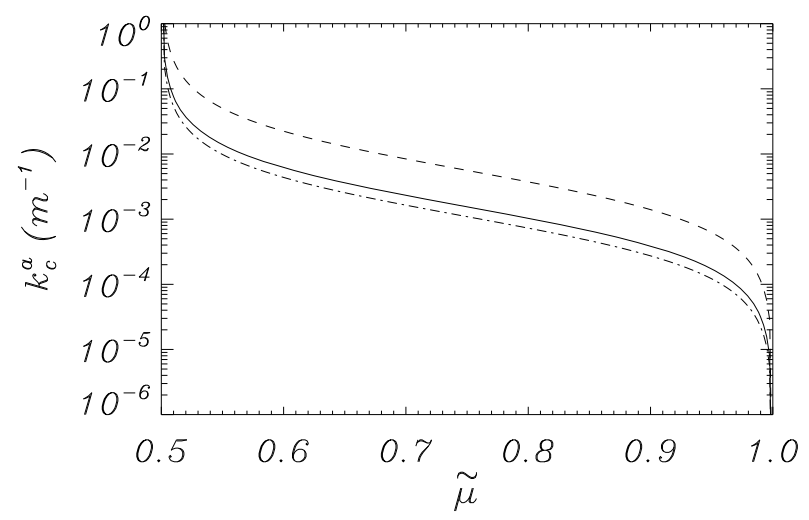

Fig. 10. Critical wavenumber versus the ionisation fraction for the Alfvén wave for different propagation angles: $\theta=0$ (dash-dotted), $\theta=\pi / 4$ (solid), and $\theta=7 \pi / 16$ (dashed).

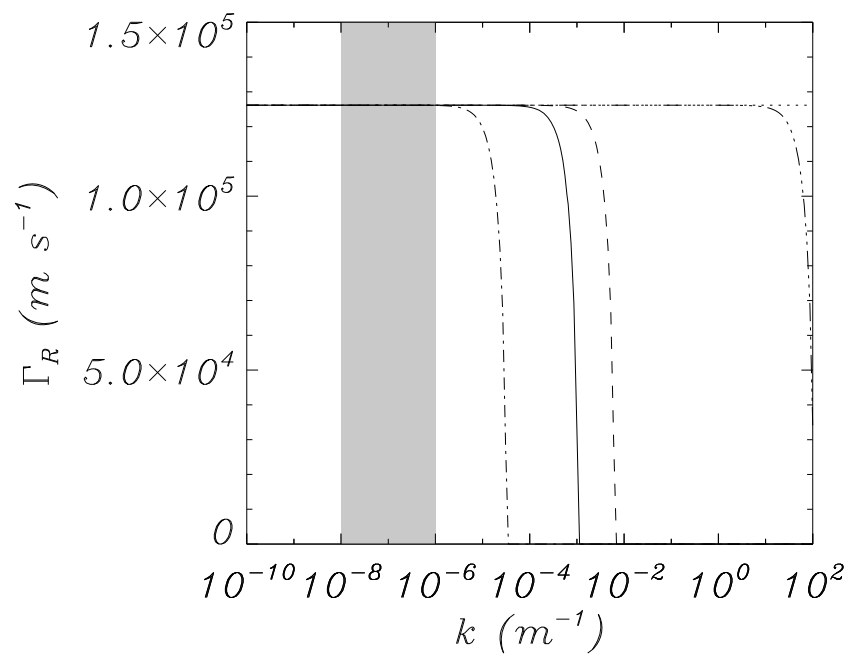

Fig. 11. Real part of the modified Alfvén speed, $\Gamma_{\mathrm{R}}$, as a function of wavenumber $\boldsymbol{k}$ in a partially ionised plasma with $\tilde{\mu}=0.5$ (dash-3dotted), $\tilde{\mu}=0.6$ (dashed), $\tilde{\mu}=0.8$ (solid), and $\tilde{\mu}=0.99$ (dash-dotted) compared with the ideal Alfvén speed, $v_{\mathrm{a}}$, (dotted). The shade region corresponds to the interval of observed wavelengths in prominences.

which is equal to 1 for a fully ionised plasma $\left(\eta_{\mathrm{C}}=\eta\right)$ and goes to infinity for $\tilde{\mu} \rightarrow 1$.

Figure 11 shows the modified Alfvén speed, Eq. (29), computed for different values of the ionisation fraction. One observes that $\Gamma_{\mathrm{R}}$ is equal to the ideal Alfvén speed, $v_{\mathrm{a}}$, for wavenumbers smaller than the critical wavenumber, $k_{\mathrm{c}}^{\mathrm{a}}$. While for wavenumbers larger than $k_{\mathrm{c}}^{\mathrm{a}}$, the modified Alfvén speed falls almost vertically to 0 , so there is no propagating wave since $\omega_{R}=0$.

\section{Conclusions}

In this paper we have studied the time damping of Alfvén and magnetoacoustic waves in a partially ionised plasma, with prominence physical conditions, considering non-adiabatic terms (thermal conduction, radiation losses and heating) in the energy equation. Small-amplitude oscillations have been assumed, so the linearised non-adiabatic one-fluid MHD equations for a partially ionised plasma have been considered and the dispersion relations for Alfvén, slow and fast magnetoacoustic waves have been found. Finally, the period and damping time of these waves has been computed. Next, we summarise the main conclusions and results of this work:

- The period of magnetoacoustic waves remains basically the same as in the ideal case. The modification to this ideal value introduced by non-adiabatic terms and ion-neutral collisions is practically negligible.

- The ion-neutral collisions mechanism is able to damp efficiently the three MHD waves (fast, slow and Alfvén) in different ways.

- From the model proposed in this work one can obtain values of the ratio of the damping time to the period similar to the ones obtained in observations, which range from 1 to 10 .

- The inclusion of non-adiabatic terms in the partially ionised set of equations increases the damping of fast and slow waves in the interval of observed wavelegths as compared with the results obtained for a non-adiabatic fully ionised plasma (Paper I) and an adiabatic partially ionised plasma (Paper II).

- For slow waves, the minima of $\tau_{\mathrm{D}} / P$, corresponding to a maximum of attenuation, are displaced to longer wavelengths as compared to when only non-adiabatic effects are considered. An increase of the neutral portion in the plasma produces a displacement of these ranges of maximum damping to longer wavelengths. Radiative losses are dominant at long wavelengths while the rest of the wavenumber interval is dominated by thermal conduction and ion-neutral collisions. For ionisation fractions with $\tilde{\mu}<0.8$ both mechanisms dominate in the considered wavelength interval (a single minimum with maximum damping caused by the combination of the two effects appears), while for $\tilde{\mu}>0.8$ the minimum splits and ion-neutral collisions dominate in the mid range interval while thermal conduction dominates at short wavelengths.

- For fast waves, radiation is the dominant damping mechanism for long wavelengths, while in the rest of the considered wavenumber interval the damping is dominated by the effect of ion neutral collisions mechanism.

- Fast waves only exist for wavenumber smaller than a critical value that depends on the ionisation fraction. In spite of 
this, the critical wavenumber is large in comparison with the typical wavenumbers of waves in prominences.

- The Alfvén wave is damped by ion-neutral collisions and is not affected by the non-adiabatic terms. The period and the damping time of Alfvén waves depends on the angle of propagation. In the case of almost neutral plasmas, the Alfvén wave is very efficiently damped.

- As with the fast wave, the Alfvén wave also presents a critical wavenumber, which depends on the ionisation fraction and the propagation angle and whose expression, in the fully ionised limit, is equal to the one obtained by Ferraro \& Plumpton (1961).

- In the case of non-adiabatic and partially ionised plasmas, the expression and behaviour of the modified sound and Alfvén speeds have been obtained.

- Finally, for typical prominence temperature values, the contribution of electrons to thermal conduction is negligible in front of the contribution of neutrals.

Acknowledgements. P. Forteza thanks the Spanish Ministerio de Educación y Ciencia for a fellowship. The authors acknowledge the financial support received from MEC AYA2006-07637. The authors also acknowledge the International Space Science Institute for the financial support received for meetings of the teams "Coronal Waves and Oscillations" and "Imaging of Quiescent and Eruptive Solar Prominences from Space" in Bern where some of the ideas in this work started to develop.

\section{References}

Ballester, J. L. 2006, Philos. Trans. R. Soc. A, 364, 405

Balthasar, H., Wiehr, E., Schleicher, H., \& Wohl, H. 1993, A\&A, 277, 635
Banerjee, D., Erdélyi, R., Oliver, R., \& O'Shea, E. 2007, Sol. Phys., 246, 3 Carbonell, M., Oliver, R., \& Ballester, J. L. 2004, A\&A, 415, 739 (Paper I) Carbonell, M., Terradas, J., Oliver, R., \& Ballester, J. L. 2006, A\&A, 460, 573 Cox, D. P., \& Tucker, W. H. 1969, ApJ, 157, 1157

Dahlburg, R. B., \& Mariska, J. T. 1988, Sol. Phys., 117, 51

Engvold, O. 2004, in Multi-Wavelength Investigations of Solar Activity, ed. A. V. Stepanov, E. E. Benevolenskaya, \& A. G. Kosovichev, IAU Symp., 223, 187 Ferraro, V. C. A., \& Plumpton, C. 1961, An introduction to magneto-fluid mechanics (Oxford University Press)

Forteza, P., Oliver, R., Ballester, J. L., \& Khodachenko, M. L. 2007, A\&A, 461, 731 (Paper II)

Foullon, C., Verwichte, E., \& Nakariakov, V. M. 2004, A\&A, 427, L5

Gilbert, H. R., Hansteen, V. H., \& Holzer, T. E. 2002, ApJ, 577, 464

Hildner, E. 1974, Sol. Phys., 35, 123

Ibáñez, S. M. H., \& Mendoza, B. C. A. 1990, Ap\&SS, 164, 193

Kendall, D. C., \& Plumpton, C. 1964, Magnetohydrodynamics with hydrodynamics, 1 (Pergamon Press)

McWhirter, R. W. P., Thonemann, P. C., \& Wilson, R. 1975, A\&A, 40, 63

Milne, A. M., Priest, E. R., \& Roberts, B. 1979, ApJ, 232, 304

Molowny-Horas, R., Wiehr, E., Balthasar, H., Oliver, R., \& Ballester, J. L. 1999, in JOSO Annu. Rep., 1998, 126, 126

Oliver, R., \& Ballester, J. L. 2002, Sol. Phys., 206, 45

Parker, E. N. 1953, ApJ, 117, 431

Patsourakos, S., \& Vial, J.-C. 2002, Sol. Phys., 208, 253

Priest, E. R. 1984, Solar magneto-hydrodynamics, Geophysics and Astrophysics Monographs (Dordrecht: Reidel)

Raymond, J. C., \& Smith, B. W. 1977, ApJS, 35, 419

Rosner, R., Tucker, W. H., \& Vaiana, G. S. 1978, ApJ, 220, 643

Soler, R., Oliver, R., \& Ballester, J. L. 2007, A\&A, 471, 1023

Terradas, J., Oliver, R., \& Ballester, J. L. 2001, A\&A, 378, 635

Terradas, J., Molowny-Horas, R., Wiehr, E., et al. 2002, A\&A, 393, 637

Terradas, J., Carbonell, M., Oliver, R., \& Ballester, J. L. 2005, A\&A, 434, 741

Tucker, W. H., \& Koren, M. 1971, ApJ, 168, 283

Wiehr, E. 2004, in SOHO 13 Waves, Oscillations and Small-Scale Transients Events in the Solar Atmosphere: Joint View from SOHO and TRACE, ed. H. Lacoste, ESA SP, 547, 185 